\title{
A análise da radiografia prévia pelo operador melhora o desempenho do localizador apical eletrônico em dentes com fratura radicular: estudo in vitro
}

\author{
The analysis of previous radiograph by the operator improves the \\ performance of the apex locator in teeth with root fracture: in vitro study
}

Tiago André Fontoura de Melo*

Renata Grazziotin Soares**

Daniele Muraro ${ }^{* * *}$

Érica Couto Prebianca**

\section{Resumo}

Objetivo: este estudo objetivou investigar se a análise da radiografia prévia pelo operador influencia no desempenho do localizador apical eletrônico MiniApex ${ }^{\circledR}$ no momento da mensuração do comprimento de trabaIho em dentes com fratura radicular apical. Materiais e método: vinte pré-molares inferiores unirradiculares foram randomicamente divididos em dois grupos: $G A$ - dentes com raiz íntegra e GB - dentes com fratura horizontal simulada no terço apical. O comprimento de trabalho foi determinado de forma visual (CTvisual) reduzindo-se $1 \mathrm{~mm}$ do comprimento total do dente (GA) ou da linha da fratura (GB). Um único operador realizou as medidas eletrônicas do comprimento de trabalho (CTeletrônico), utilizando a marca de 0.5 no display do aparelho. Para ambos os grupos, as medidas eletrônicas foram realizadas em fases distintas: fase I sem análise da radiografia prévia e fase II - com análise da radiografia prévia (medindo-se com régua milimetrada o comprimento aparente de trabalho). O desempenho do localizador apical foi investigado por meio da comparação de cada medida do CTeletrônico com o CTvisual; utilizando-se, para análise dos dados, o teste $t$ de Student. Resultados: para o GA, não houve diferença estatística em relação ao desempenho do localizador tanto para a fase $I(p=0,619)$ como para a fase $I I(p=$ $0,177)$. Para o $G B$ houve diferença estatística para a fase $I(p=0,003)$, mas não para a fase II $(p=0,489)$. Conclusão: a análise da radiografia prévia pelo operador, fator indispensável na prática clínica, melhorou o desempenho do localizador eletrônico apical MiniApex ${ }^{\circledR}$ durante a mensuração do comprimento de trabalho em dentes extraídos com fratura radicular apical simulada.

Palavras-chave: Endodontia. Diagnóstico. Fraturas dos dentes. Radiografia. Odontometria.

\section{Introdução}

A análise da radiografia prévia é fundamental para se realizar um correto diagnóstico e plano de tratamento em endodontia ${ }^{1-3}$, evitando erros operatórios durante o tratamento. Os exames radiográficos auxiliam ao longo de todas as fases do preparo químico mecânico e, evidentemente, na mensuração do comprimento de trabalho. No decorrer da reali-

\footnotetext{
Mestre em Endodontia. Professor do curso de Odontologia da Faculdade da Serra Gaúcha, Caxias do Sul, RS, Brasil.

Doutora em Endodontia. Professora do curso de Odontologia da Universidade Federal do Rio Grande do Sul, Porto Alegre, RS, Brasil.

-* Especialista em Endodontia, Porto Alegre, RS, Brasil.
} 
zação da odontometria convencional pela técnica de Ingle ${ }^{4}$, são necessárias, em média, duas radiografias periapicais para a realização da medida.

Mais recentemente, com a disseminação do uso clínico dos aparelhos localizadores apicais eletrônicos, a necessidade de realização de tomadas radiográficas durante a etapa de mensuração do comprimento de trabalho endodôntico tem sido minimizada, ou até dispensada. Inúmeros estudos científicos vêm mostrando a adequada acurácia dos localizadores apicais eletrônicos, no que concerne à realização da medida do comprimento de trabalho endodôntico, tanto em modelos in vitro ${ }^{5-8}$, quanto in $v_{i v o}{ }^{9,10}$. Dessa maneira, a realização do exame radiográfico como coadjuvante ao uso do localizador apical na mensuração do comprimento de trabalho é desnecessária. Em contrapartida, autores indicam a tomada radiográfica durante a fase de odontometria eletrônica quando o operador necessita de mais informações em relação à anatomia, bem como em situações específicas, nas quais, por diferentes motivos, a medida permanece não confiável ${ }^{11}$.

Apesar do exposto, a radiografia prévia à realização do tratamento endodôntico é indispensável, sendo um consenso entre os autores ${ }^{3,12,13}$. Essa tomada radiográfica permite ao operador verificar detalhes sobre a anatomia radicular e o comprimento aparente do dente, informações que possibilitam antever se o tratamento ocorrerá normalmente ou com alguma intercorrência. Em dentes traumatizados, por exemplo, que apresentam fratura radicular apical, a radiografia prévia possibilita vislumbrar um comprimento adequado de trabalho, aquém da linha de fratura, objetivando a manutenção da vitalidade do segmento apical ${ }^{14-16}$.

Assim, durante a utilização do localizador apical eletrônico, a análise da radiografia prévia acrescenta informações ao operador. Essas informações teóricas podem servir como base para um aumento de sinestesia quando se utiliza o localizador apical eletrônico, o que poderia influenciar no desempenho do aparelho. Dessa maneira, este estudo investigou se a análise da radiografia prévia pelo operador influencia no desempenho do localizador apical eletrônico MiniApex ${ }^{\circledR}$ no momento da mensuração do comprimento de trabalho em dentes com fratura radicular apical.

\section{Materiais e método}

Este estudo foi aprovado pelo Comitê de Ética em Pesquisa da Universidade de Passo Fundo (Protocolo $\mathrm{n}^{\mathrm{o}}$ 10755612.0.0000.5342) previamente à sua realização. Utilizaram-se 20 pré-molares inferiores unirradiculares humanos extraídos por motivos diversos, fornecidos pelo Banco de Dentes Humanos da mesma instituição. Os dentes apresentavam comprimento e anatomia semelhantes, verificados pelo exame visual. Os critérios de exclusão foram observados por meio do exame radiográfico: tratamento endodôntico prévio, presença de retentor intrarradicular, reabsorções, calcificações, dilacerações e fratura radicular.

\section{Preparo dos dentes}

Um operador realizou abertura coronária e esvaziamento com limas tipo K \#10 [Dentsply/Maillefer $\left.{ }^{\circledR}\right]$ e solução de hipoclorito de sódio a $2,5 \%$. Os dentes foram divididos de forma aleatória. Primeiramente, os dentes foram inseridos em um envelope opaco e, subsequentemente, removidos, um por vez, em dois grupos de dez. No GA $(n=10)$, os dentes permaneceram com a raiz íntegra. No GB $(n=10)$, foi confeccionada uma canaleta, por meio de um disco de carburundum, perpendicular ao longo eixo da raiz, a aproximadamente $3 \mathrm{~mm}$ do ápice. Essa canaleta objetivou simular uma fratura radicular apical incompleta. Para tanto, foi confeccionada na direção vestibular para lingual, abrangendo $2 / 3$ do diâmetro da secção transversal naquele local.

\section{Radiografias}

Após preparo, cada dente foi fixado na superfície de um filme radiográfico (AGFA DENTUS M2 Comfort). As tomadas foram realizadas com o aparelho radiográfico (TIMEX 70C - Gnatus ${ }^{\circledR}$ ), operando em $70 \mathrm{kv}, 08 \mathrm{~mA}$ e 0,20 segundos de exposição, posicionado de maneira que o raio central incidisse perpendicularmente ao filme radiográfico com uma distância focal de $40 \mathrm{~cm}$. Essa etapa foi padronizada por meio da utilização de uma plataforma previamente confeccionada.

\section{Determinação do comprimento de trabalho visual (CTvisual)}

Com o canal radicular repleto de hipoclorito de sódio, inseriu-se uma lima tipo K \#10 (Dentsply/ Maillefer ${ }^{\circledR}$ ) até que sua ponta ficasse visível no forame apical (para o GA), ou na linha da canaleta (para o GB). Ajustou-se o cursor de borracha no ponto de referência e obteve-se o comprimento do dente (para o GA), ou o comprimento até a linha da canaleta (para o GB). O CTvisual foi determinado diminuindo-se $1 \mathrm{~mm}$ do comprimento total.

\section{Determinação do comprimento de traballho eletrônico (CTeletrônico)}

\section{- Fase I:}

Os dentes foram fixados em recipientes plásticos contendo alginato (Jeltrate-Dentsply ${ }^{\circledR}$ ) com a porção radicular submersa no material. O canal radicular foi preenchido com hipoclorito de sódio a 
2,5\% até o terço cervical, deixando a câmara pulpar isenta de solução. Um único operador experiente na utilização do localizador apical MiniApex ${ }^{\circledR}$ (SybronEndo) realizou as medidas, utilizando a marca de 0.5 no display do aparelho ${ }^{17}$, empregando limas tipo $\mathrm{K}$ compatíveis com o tamanho do canal radicular (\#15, \#20 ou \#25 (Dentsply/Maillefer ${ }^{\circledR}$ ). A alça labial do aparelho foi inserida no alginato.

\section{Fase II:}

Em outro momento, o mesmo operador realizou exatamente os mesmos procedimentos descritos na fase I, porém tendo analisado previamente a radiografia. Assim, foi possível verificar a anatomia radiográfica do dente e medir, com régua endodôntica milimetrada, o comprimento do dente (para o GA), ou o comprimento até a canaleta (para o GB).

\section{Análise dos dados}

O desempenho do localizador apical foi investigado por meio da comparação de cada medida do CTeletrônico (fase I e fase II) com o CTvisual, utilizando-se, para análise dos dados, o teste $\mathrm{t}$ de Student. A influência da análise da radiografia prévia no desempenho do localizador apical foi dicotomizada: 1) melhora o desempenho: quando não houve diferença estatística entre os valores médios do CTvisual e do CTeletrônico ( $\mathrm{p}>0,05)$, ou 2) não influencia no desempenho: quando houve diferença estatística entre os valores médios do CTvisual e do CTeletrônico $(\mathrm{p}<0,05)$.

\section{Resultados}

A Tabela 1 mostra os valores médios $(\mathrm{mm})$ e desvio-padrão obtidos durante as mensurações do CTvisual e do CTeletrônico (fases I e II) verificadas para os dentes dos dois grupos.

Para o GA (raiz íntegra) não houve diferença estatística em relação ao desempenho do localizador tanto para a fase I $(p=0,619)$ como para a fase II $(\mathrm{p}=0,177)$. Para o GB (fratura radicular simulada) houve diferença estatística para a fase I $(p=0,003)$, mas não para a fase II $(p=0,489)$.

Tabela 1 - Média ( $\mathrm{mm}$ ) e desvio-padrão do comprimento de trabalho visual (CTvisual) e do comprimento de trabalho eletrônico (CTeletrônico fases I e II) obtidos para os dentes dos dois grupos (teste T de Student $p<0,05$ )

\begin{tabular}{|c|c|c|c|c|c|}
\hline Grupo & $\mathrm{n}$ & & & $\begin{array}{l}\text { Medida do comprimento de trabalho } \\
(\mathrm{mm}) \text { e desvio-padrão }\end{array}$ & $p$ \\
\hline \multirow{3}{*}{ GA } & \multirow{3}{*}{10} & \multicolumn{2}{|l|}{ CTvisual } & $19,90(1,91)$ & \\
\hline & & CT & Fase I & $19,80(1,85)$ & 0,619 \\
\hline & & Cleletrônıco & Fase II & $19,65(1,73)$ & 0,177 \\
\hline \multirow{3}{*}{ GB } & \multirow{3}{*}{10} & CTvisual & & $15,10(1,26)$ & \\
\hline & & \multirow{2}{*}{ CTeletrônico } & Fase I & $17,00(1,31)$ & $0,003^{*}$ \\
\hline & & & Fase II & $15,40(1,82)$ & 0,489 \\
\hline
\end{tabular}

Teste T de Student, $\alpha=0,05 \%$, " estatisticamente significante

\section{Discussão}

A importância da radiografia prévia ao tratamento endodôntico em dentes com fratura radicular apical e o desafio de um adequado manejo dos localizadores apicais eletrônicos, objetivando precisão na medida do comprimento de trabalho, foram os temas que impulsionaram esta investigação. Os resultados deste estudo in vitro mostraram que a análise da radiografia prévia pelo operador, possibilitando medir o comprimento aparente do dente (ou do fragmento coronário, em casos de fratura), melhorou o desempenho do localizador eletrônico apical MiniApex ${ }^{\circledR}$ durante a mensuração do comprimento de trabalho em dentes extraídos com fratura radicular apical simulada.

Embora as radiografias periapicais forneçam uma imagem bidimensional do sistema de canais, que é uma estrutura tridimensional, a sua interpretação permite observar detalhes da anatomia radicular interna e externa ${ }^{18}$. Alguns autores sugerem que duas radiografias periapicais de diagnóstico deveriam ser realizadas, por meio da técnica de dissociação radiográfica horizontal, para possibilitar uma cuidadosa avaliação da morfologia do sistema de canais ${ }^{13}$.

No presente estudo, o protocolo empregado para a realização da área de fratura horizontal incompleta junto à porção apical da raiz dentária foi realizado conforme evidências disponíveis na publicação de Azabal et al. ${ }^{19}$ (2004). Tais injúrias à raiz comprometem o padrão anatômico normal, o que pode interferir na sinestesia quando da utilização do localizador apical eletrônico para mensuração do comprimento de trabalho, que deve permanecer aquém do fragmento fraturado. 
Nessa linha, os resultados deste estudo mostraram que a minuciosa análise da radiografia prévia, com mensuração do comprimento aparente de trabalho, funciona como coadjuvante durante as manobras de utilização do localizador apical MiniApex ${ }^{\circledR}$ em dentes com fratura apical simulada, melhorando o seu desempenho. Uma possível explicação para a melhora no desempenho do aparelho está no aumento da sinestesia obtido pelo operador, que se torna ciente do possível comprimento de trabalho a ser utilizado, parâmetro fornecido pela radiografia prévia.

Interessante notar que, nos dentes com raízes íntegras (GA), a análise da radiografia prévia não teve influência no desempenho do aparelho, o que foi evidenciado pela ausência de diferença estatística (Tabela 1) entre as mensurações do CTvisual e do CTeletrônico (tanto na fase I, como na fase II). A hipótese que tenta justificar esse achado é que, em dentes com raízes íntegras, o operador tem uma maior sensibilidade/sinestesia, mesmo sem a utilização de uma radiografia prévia. Isso ocorre em virtude do funcionamento dos localizadores apicais eletrônicos que fecham o circuito quando há o estreitamento no canal radicular, ou seja, o valor de impedância medido se equipara ao da mucosa labial, local onde está conectada a alça labial ${ }^{20}$. É importante lembrar que o operador, durante a utilização do MIniApex ${ }^{\oplus}$ na fase I, não sabia se o dente apresentava raiz íntegra ou fraturada.

Em virtude dos diferentes desenhos metodológicos, a literatura já demonstrou precisão na aferição de fraturas radiculares horizontais com o uso dos localizadores apicais ${ }^{19,21}$, bem como imprecisão na detecção de áreas de fratura radicular ${ }^{22,23}$.

É importante salientar que os resultados aqui encontrados não podem ser extrapolados de uma maneira direta para a prática clínica, em virtude dos diversos fatores implicados na mensuração do comprimento de trabalho endodôntico de dentes fraturados em pacientes, tais como o envolvimento combinado de outros tipos de traumatismo, como fratura alveolar, por exemplo, além das características anatômicas próprias do elemento dentário: atresia, calcificação, reabsorção, entre outras.

Assim, à luz das limitações deste estudo in vitro, é possível concluir que análise da radiografia prévia pelo operador, fator indispensável na prática clínica, melhorou o desempenho do localizador eletrônico apical MiniApex ${ }^{\circledast}$ durante a mensuração do comprimento de trabalho em dentes extraídos com fratura radicular apical simulada.

\section{Albstract}

Objective: this study investigated whether the analysis of previous radiograph by the operator influences the performance of the electronic apex locator MiniApex ${ }^{\circledR}$, upon measuring the working length in teeth with api- cal root fracture. Materials and method: twenty singlerooted lower premolars were randomly allocated in two groups: $G A$ - teeth with intact root, and $G B$ - teeth with simulated horizontal fracture on the apical third. The working length was determined by visual examination (visual WL) $1 \mathrm{~mm}$ shorter from either the total tooth length $(G A)$ or the fracture line $(G B)$. A single operator performed the working length electronic measures (electronic WL) using the 0.5 mark on the device's display. For both groups, the electronic measures were performed in two different moments: phase I - without analysis of previous radiograph, and phase II - with analysis of previous radiograph (measuring the apparent WL with a millimeter ruler). The performance of the apex locator was investigated by comparing each electronic $W L$ measurement to the visual $W L$, using the Student's $t$ test for data analysis. Results: there was not statistical difference in the GA group regarding the performance of the apex locator for phase I ( $p=0.619)$, as well as for phase II $(p=0.177)$. There was statistical difference in the GB group for phase I ( $p=0.003)$, but not for phase II ( $p=0.489)$. Conclusion: The analysis of previous radiograph by the operator is imperative for clinical practice, and it improved the performance of the electronic apex locator MiniApex ${ }^{\circledast}$ during the measurement of the WL in extracted teeth with simulated apical root fracture.

Keywords: Endodontics. Diagnosis. Tooth fractures. Radiograph. Odontometry.

\section{Referências}

1. Gutmann JL, Leonard JE. Problem solving in endodontic working-length determination. Compend Contin Educ Dent $1995 ; 16(3): 288-94$

2. Marques JLL, Fenyo-Pereira M, Safioti LML. Análise radiográfica da qualidade do tratamento endodôntico e suas interações. Rev Bras Odontol 1996; 53(3):11-5.

3. Cantatore G, Berutti E, Castellucci A. Missed anatomy: frequency and clinical impact. Endodontic Topics 2009; 15(1):3-31.

4. Bramante CM, Berbert A. A critical evaluation of some methods of determining tooth length. Oral Surg Oral Med Oral Pathol Oral Radiol Endod 1974; 37(3):463-73.

5. Goldberg F, Marroquín BB, Frajlich S, Dreyer C. In vitro evaluation of the ability of three apex locators to determine the working length during retreatment. J Endod 2005; 31(9):676-8.

6. D’Assunção FL, Albuquerque DS, Queiroz Ferreira LC. The ability of two apex locators to locate the apical foramen: an in vitro study. J Endod 2006; 32(6):560-2.

7. Goldberg F, Frajlich S, Kuttler S, Manzur E, Briseño-Marroquín $\mathrm{B}$. The evaluation of four electronic apex locators in teeth with simulated horizontal oblique root fractures. J En$\operatorname{dod} 2008$; 34(12):1497-9

8. D’Assunção FL, Albuquerque DS, Salazar-Silva JR, Dos Santos VC, Sousa JC. Ex vivo evaluation of the accuracy and coefficient of repeatability of three electronic apex locators using a simple mounting model: a preliminary report. Int Endod J 2010; 43(4):269-74. 
9. Chevalier V, Arbab-Chirani R, Nicolas M, Morin V. Occurrence of no-function of two electronic apex locators: an in vivo study. Oral Surg Oral Med Oral Pathol Oral Radiol Endod 2009; 108(6):61-5.

10. Renner D, Grazziotin-Soares R, Gavini G, Barletta FB. Influence of pulp condition on the accuracy of an electronic foramen locator in posterior teeth: an in vivo study. Braz Oral Res 2012; 26(2):106-11.

11. Lopes HP, Siqueira Jr. JF. Endodontia Biologia e Técnica. 3. ed. Rio de Janeiro: Guanabara Koogan; 2010.

12. Nattress BR, Martin DM. Predictability of radiographic diagnosis of variations in root canal anatomy in mandibular incisor and premolar teeth. Int Endod J 1991; 24(2):58-62.

13. Castellucci A. Endodontic radiography. In: Castellucci A. Endodontics. 2. ed. Florence: Tridente; 2006. v. 1. p. 66-119.

14. Malhotra N, Kundabala M, Acharaya S. A review of root fractures: diagnosis, treatment and prognosis. Dent Update 2011; 38(9):615-624.

15. Brito-Júnior M, Camilo CC, Soares JA, Souza LN, Moreira-Júnior G, Faria e Silva AL. Endodontic management of a long-standing horizontal mid-root fracture: case report in a young patient. Pediatr Dent 2012; 34(3):69-71.

16. May JJ, Cohenca N, Peters OA. Contemporary management of horizontal root fractures to the permanent dentition: diagnosis-radiologic assessment to include cone-beam computed tomography. Pediatr Dent 2013; 35(2):120-4.

17. Akisue E, Gratieri SD, Barletta FB, Caldeira CL, Grazziotin-Soares R, Gavini G. Not all electronic foramen locators are accurate in teeth with enlarged apical foramina: an in vitro comparison of 5 brands. J Endod 2014; 40(1):109-12.

18. Slowey RR. Root canal anatomy. Road map to successful endodontics. Dent Clin North Am 1979; 23(4):555-73.

19. Azabal M, Garcia-Otero D, Macorra JC. Accuracy of the Justy II apex locator in determining working length in simulated horizontal and vertical fractures. Int Endod J 2004; 37(3):174-7.

20. Nekoofar MH. Electronic apex locators. Int Endod J 2005 Jun; 38(6):417-8.

21. Ebrahim AK, Wadachi R, Suda H. Accuracy of three different electronic apex locators in detecting simulated horizontal and vertical root fractures. Aust Endod J 2006; 32(2):64-9.

22. Zhang P. An in vitro study of two kinds of root apex locators to diagnose vertical root fracture. Hua Xi Kou Qiang Yi Xue Za Zhi 2009; 27(5):528-30.

23. Beri L, Matariya G. The evaluation of three electronic apex locators in teeth with simulated incomplete oblique root fractures. J Int Clin Dent Res Organ 2009; 3(1):49-54.

\section{Endereço para correspondência:}

Tiago André Fontoura de Melo Rua Eça de Queiróz, 466/701, Petrópolis 90670-020 Porto Alegre/RS Fone: (51) 84035644

E-mail: tafmelo@gmail.com

Recebido: 10/08/2013. Aceito: 21/01/2014. 